ORIGINAL ARTICLE

\title{
Acanthosis nigricans: relation with type 2 diabetes mellitus, anthropometric variables, and body mass in Indians
}

\author{
N P Grandhe, A Bhansali, S Dogra, B Kumar
}

Postgrad Med J 2005;81:541-544. doi: 10.1136/pgmj.2004.028308

See end of article for
authors' affiliations
$\ldots \ldots \ldots \ldots \ldots \ldots . . . . . . .$.
Correspondence to:
Dr A Bhansali, Department
of Endocrinology,
Postgraduate Institute of
Medical Education and
Research, Chandigarh160
012 , India;
anilbhansali_endocrine@
rediffmail.com
Submitted
1 September 2004
Accepted
26 November 2004
Objective: To determine the prevalence of acanthosis nigricans (AN) in type 2 diabetes mellitus (T2DM) and its correlation with various anthropometric measurements in Indians.

Methods: One hundred and fifty consecutive subjects with T2DM attending the diabetes clinic at a tertiary referral centre in North India were considered as cases and 150 age and sex matched healthy attendants of non-diabetic subjects as controls. All the cases and controls were screened for the presence of AN and its severity. Anthropometric measurements of all of them were measured in standard method. Regression analysis was done to determine the association of AN with T2DM and various anthropometric measurements.

Results: The prevalence of AN in subjects with diabetes and healthy controls was $62.6 \%$ and $40 \%$ respectively, and this difference was significant $(p<0.05)$. Body mass index (BMI) between cases and controls was comparable by chance. There was a statistically significant correlation of increasing severity of AN with increasing BMI, waist circumference, hip circumference, waist-hip ratio, skinfold thickness, and body fat percentage in diabetic patients. However, in regression analysis after considering all the confounding factors there was a significant correlation of AN, only with diabetes mellitus and BMI.

Conclusions: Indians have high prevalence of $\mathrm{AN}$ and it is an independent cutaneous marker of both T2DM and BMI.
$\mathrm{T}$ he prevalence of diabetes and its adverse health effects has risen most rapidly in South Asia. India is becoming a "diabetes capital" having a higher number of people with diabetes than any other country, with estimates ranging from 19.4 million in $1995^{1}$ to 32.7 million in $2000 .^{2}$ This number is expected to rise by $195 \%$ in India during 1995-2025 to reach 57.2 million in $2025 .^{1}$

The association of hyperinsulinaemia, insulin resistance, and obesity with acanthosis nigricans (AN) has been of increasing interest to doctors. ${ }^{3}$ The prevalence of AN in the literature varies from $7 \%$ in an unselected population to $74 \%$ in obese persons, ${ }^{4}{ }^{5}$ whereas little information is available on its prevalence in adult diabetic subjects. ${ }^{6}$ To the best of our knowledge there are no studies showing the prevalence of AN in type 2 diabetes mellitus (T2DM) and its true correlation with various anthropometric measurements in Indians.

\section{METHODS}

One hundred and fifty subjects with T2DM diagnosed according to the World Health Organisation criteria ${ }^{7}$ attending the diabetes clinic of Nehru Hospital, PGIMER, Chandigarh, were randomly selected for this study. Similar number of age and sex matched healthy attendants of nondiabetic patients were randomly taken as controls after screening for T2DM. People with associated other endocrine and systemic diseases known to result in AN were excluded from the study. Subjects with history of intake of drugs including nicotinic acid, oral contraceptives, and application of topical fusidic acid, ${ }^{8-12}$ which can cause AN were excluded from this study.

Weight, height, waist and hip circumferences (WC, HC), waist hip ratio (WHR), and skinfold thickness (SFT) were obtained in a standardised technique by a single observer. Waist circumference (midway between iliac crest and lower margin of ribs) and hip circumference (maximum circumference of buttocks) were measured by using a flexible nonelastic tape whereas SFT was measured with the standard

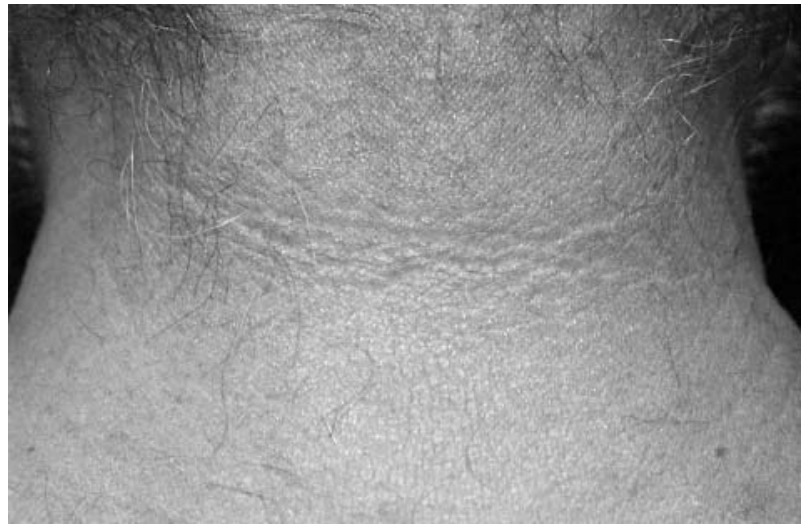

Figure 1 Grade 1 acanthosis nigricans.

Lange caliper (John Bull, British Indicators Limited) at four sites (biceps, triceps, sub-scapular, and supra-iliac) on nondominant side of the body. All the readings were measured three times and the average of the three readings was taken as final. The body mass index (BMI) was calculated by weight in kilograms divided by height in metres squared. A BMI of $\geqslant 23 \mathrm{~kg} / \mathrm{m}^{2}$ was considered as overweight for both men and women. ${ }^{13}$ All the subjects were examined for presence of AN at different sites by a dermatologist and its presence was confirmed by a second observer. The severity grading of AN was done using a standard scale of $0-4$ as described by Burke et al. ${ }^{6}$ According to this scale grading at neck was described as grade 0 : if it is not visible on close

Abbreviations: AN, acanthosis nigricans; T2DM, type 2 diabetes mellitus; $\mathrm{BMI}$, body mass index; WHR, waist-hip ratio; SFT, skinfold thickness; WC, waist circumference; $\mathrm{HC}$, hip circumference; IP, impedence plethysmograph 


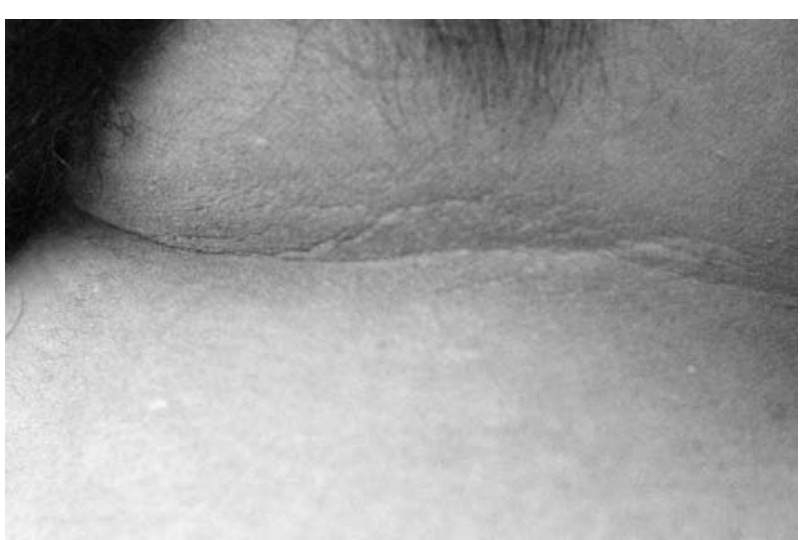

Figure 2 Grade 2 acanthosis nigricans.

inspection, grade 1 : clearly present on close visual inspection, extent not measurable (fig 1 ), grade 2 : limited to base of the skull, does not extend to the lateral margins of the neck (fig 2), grade 3: extending to the lateral margins of the neck, but not visible from front (fig 3), and grade 4: extending anteriorly. Body fat percentage was measured using impedance plethysmograph (IP) (OMRON BF 302(HBF-302-E), Omran Matsusaka, Japan) by a standard technique, which reflects the combined visceral and subcutaneous fat composition. Body fat measurement by IP indirectly reflects visceral fat composition. The body fat percentage was also calculated from SFT using the equation of $\mathrm{Siri}^{14}$ and it was compared with body fat percentage calculated from the IP.

\section{Statistical methods}

Statistical analysis was performed using Microsoft Excel 2002 and the Statistical Package for the Social Sciences (SPSS) for Windows, release 10 (SPSS, Chicago, IL). Results were expressed as mean (SD) unless otherwise specified. Baseline characteristics were compared using the $\chi^{2}$ test for qualitative (categorical) variables and Student's $t$ test for quantitative (continuous) variables. To compare continuous variables among more than two groups, one way analysis of variance was used ( $F$ test) and post-hoc tests were performed using the Bonferroni method. Spearman's correlation coefficient was used to analyse the association between two continuous variables. Regression analysis was done to study the factors affecting AN. A p value of $<0.05$ was considered as significant.

\section{RESULTS}

Table 1 shows the demographic and anthropometric variables of cases and controls. The mean age, BMI, SFT, and body fat percentage calculated from SFT showed no significant differences between cases and controls. However, diabetic patients had significantly higher WC, WHR, and body fat

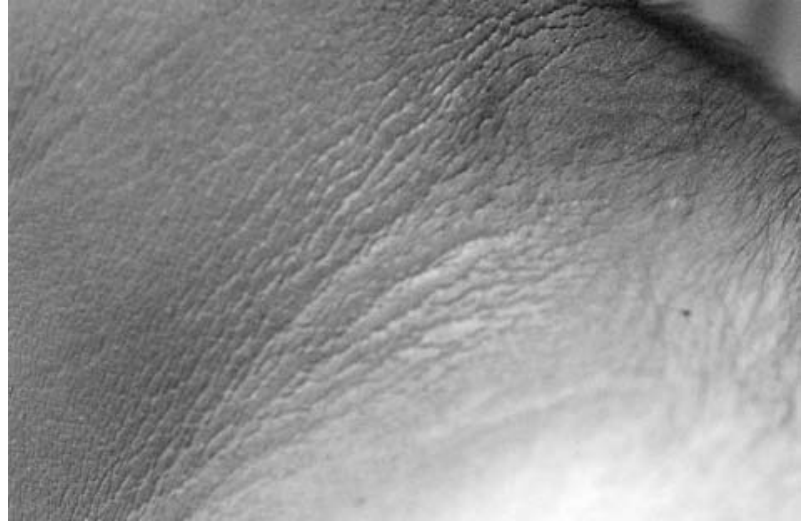

Figure 3 Grade 3 acanthosis nigricans.

percentage calculated from IP. Furthermore, there was a significant difference between body fat percentages calculated from SFT and from IP in both groups and this incongruence was more in diabetic patients. Table 2 shows the various anthropometric and body composition factors in subjects with or without AN. There was a significant difference between the prevalence of AN in diabetic patients $(62.6 \%)$ and healthy controls $(40 \%)(p<0.05)$. Among 111 overweight diabetic patients, $80(72 \%)$ had AN, whereas of 77 overweight healthy subjects $37(52.2 \%)$ had AN. No significant difference in the prevalence of AN was seen with regard to age and sex in both the groups, and duration of T2DM in cases. Those with AN in both the groups had significantly higher BMI, WC, HC, WHR, body fat percentages calculated from SFT and IP. Nape of the neck was the most frequently affected site for AN in both the cases as well as controls $(93.5 \%)$, followed by axilla $(40 \%)$, popliteal fossa $(9.7 \%)$, cubital fossa $(7.1 \%)$, knuckles $(6.5 \%)$, and other areas (3.9\%) being groin, dorsum of toes, flanks, retro-auricular areas, and naso-labial folds.

The anthropometric and body composition factors were evaluated by degree of severity of AN in both diabetic and healthy subjects (table 3 ). Among 94 diabetic subjects with AN $(62.6 \%)$, grade 1 , grade 2 , and grade 3 AN was seen in 48 $(51.1 \%), 33(35.1 \%)$, and $13(13.8 \%)$ patients respectively. While among the healthy subjects with AN, none had grade 3 A and AN of grade 1 and grade 2 severity was seen in were 40 $(66.7 \%)$ and $20(33.3 \%)$ respectively. There was a statistically significant correlation with increase in BMI, WC, HC, WHR, SFT, and body fat percentage calculated from SFT and IP with increasing degree of severity of AN in diabetic patients, whereas such relation was seen only with BMI, HC, and SFT in healthy subjects. However, the increase of these parameters with degree of severity of AN was also not uniform in diabetic subjects. Significant difference was seen in the above variables (except WHR) in diabetes patients with AN of grade

Table 1 Demographic and anthropometric variables of cases and controls

\begin{tabular}{llll}
\hline Variable & Cases $(\mathbf{n}=150)$ & Controls $(\mathbf{n}=150)$ & p Value \\
\hline Age $(\mathrm{y})$ & $52.2(10.8)$ & $49.9(11.6)$ & 0.077 \\
BMl & $25.7(4.2)$ & $25.3(4.2)$ & 0.476 \\
WC (cm) & $90.9(11.08$ & $86.2(10.3)$ & $0.001^{*}$ \\
HC (cm) & $94.5(9.3)$ & $96.8(8.5)$ & $0.024^{*}$ \\
WHR & $0.96(0.07)$ & $0.89(0.1)$ & $0.000^{*}$ \\
SFT (mm) & $72.6(31.2)$ & $71.1(30.1)$ & 0.808 \\
Body fat \% (SFT) & $25.5(6.3)$ & $25.4(6.6)$ & 0.905 \\
Body fat \% (IP) & $29.5(9.1)$ & $27.4(8.5)$ & $0.041^{*}$ \\
\hline
\end{tabular}

BMI, body mass index; HC, hip circumference; WC, waist circumference; WHR, waist-hip circumference; SFT, skinfold thickness; IP, impedance plethysmograph. * $\mathrm{p}$ Value is significant. 
Table 2 Demographic and anthropometric factors in diabetic and healthy subjects with or without acanthosis nigricans

\begin{tabular}{|c|c|c|c|c|c|c|}
\hline \multirow[b]{2}{*}{ Variable } & \multicolumn{2}{|c|}{ Acanthosis nigricans (diabetic subjects $/ \mathrm{n}=150$ ) } & \multirow[b]{2}{*}{$\mathrm{p}$ Value } & \multicolumn{2}{|c|}{$\begin{array}{l}\text { Acanthosis nigricans (non- diabetic subjects/ } \\
n=150 \text { ) }\end{array}$} & \multirow[b]{2}{*}{ p Value } \\
\hline & Present $n=94(62.6 \%)$ & Absent $n=58(37.4 \%)$ & & Present $n=60(40 \%)$ & Absent $n=90(60 \%)$ & \\
\hline Age & $52.2(10.4)$ & $52.1(11.5)$ & 0.960 & $51.2(11.6)$ & 49.1 (11.5) & 0.287 \\
\hline \multirow[t]{2}{*}{ Sex } & M:50 $(62.5 \%)$ & M:30 (37.5\%) & 0.964 & M:28 (35\%) & M:52 (65\%) & 0.181 \\
\hline & F: 44 (62.9\%) & F:26 (37.1\%) & & F:32 (45.7\%) & F:38 (44.3\%) & \\
\hline Duration $\dagger$ & $8.7(7.3)$ & $9.1(7.0)$ & 0.787 & & & \\
\hline BMI & $27.3(4.1)$ & $22.9(2.7)$ & $0.000^{*}$ & $27.9(3.5)$ & $23.6(3.7)$ & $0.000^{*}$ \\
\hline$W C(\mathrm{~cm})$ & 94.8 (10.9) & $84.5(8.0)$ & $0.000^{*}$ & $92.5(9.9)$ & $82.0(13.3)$ & $0.000^{*}$ \\
\hline WHR & $0.98(0.1)$ & $0.94(0.1)$ & $0.002^{*}$ & $0.92(0.1)$ & $0.87(0.1)$ & $0.000^{*}$ \\
\hline SFT(mm) & $81.7(31.2)$ & $56.9(23.1)$ & $0.000^{*}$ & $88.3(30.2)$ & $60.7(24.7)$ & $0.000^{*}$ \\
\hline Body fat\% (SFT) & $27.5(5.75)$ & $22.1(5.8)$ & $0.000^{*}$ & $28.8(5.7)$ & $23.2(6.2)$ & $0.000^{*}$ \\
\hline Body fat\% (IP) & $31.2(9.1)$ & $26.7(8.4)$ & $0.003^{*}$ & $31.5(7.9)$ & $24.8(8.0)$ & $0.000^{*}$ \\
\hline
\end{tabular}

Table 3 Anthropometric variables and body composition factors with degree of severity of $\mathrm{AN}$ in diabetic subjects and controls

\begin{tabular}{|c|c|c|c|c|c|c|c|}
\hline \multirow[b]{2}{*}{ Variable } & \multicolumn{3}{|c|}{ AN in diabetic subjects } & \multirow[b]{2}{*}{ p Value } & \multicolumn{2}{|c|}{ AN in controls } & \multirow[b]{2}{*}{ p Value } \\
\hline & $\begin{array}{l}\text { Grade } 1 \\
(n=48) \\
(51.1 \%)\end{array}$ & $\begin{array}{l}\text { Grade } 2 \\
(n=33) \\
(35.1 \%)\end{array}$ & $\begin{array}{l}\text { Grade } 3 \\
(n=13) \\
(13.8 \%)\end{array}$ & & $\begin{array}{l}\text { Grade } 1 \\
(n=40) \\
(66.7 \%)\end{array}$ & $\begin{array}{l}\text { Grade } 2 \\
(n=20) \\
(33.3 \%)\end{array}$ & \\
\hline BMI & $26.4(3.9)$ & $27.0(3.6)$ & $32.0(3.9) \dagger$ & $0.000^{*}$ & $26.9(3.3)$ & $30.0(3.0)$ & $0.001^{*}$ \\
\hline SFT & $76.2(26.7)$ & 79.3 (33.7) & $112.1(27.6) \dagger$ & $0.003^{*}$ & $82.3(29.3)$ & $100.3(29.0)$ & $0.029^{*}$ \\
\hline $\begin{array}{l}\text { Body fat \% } \\
\text { (SFT) }\end{array}$ & $26.7(5.4)$ & $27.0(6.1)$ & $32.7(3.8) \dagger$ & $0.007^{*}$ & $27.8(7.5)$ & $30.7(5.9)$ & 0.061 \\
\hline $\begin{array}{l}\text { Body fat \% } \\
\text { (IP) }\end{array}$ & $31.5(8.6)$ & $28.0(9.6)$ & $39.1(5.6) \dagger$ & $0.002^{*}$ & $30.9(7.8)$ & $32.6(8.1)$ & 0.436 \\
\hline
\end{tabular}

2 and grade 3 but not between those with grade 1 and grade 2. In the regression analysis after considering all the confounding factors (BMI, WC, HC, WHR, SFT, and body fat percentage), there was a significant correlation of $\mathrm{AN}$ with only diabetes mellitus and BMI.

\section{DISCUSSION}

The burden of disease in South Asia is changing. Changes in lifestyle and work patterns resulting in less physical activity and more obesity have coincided with a surge in consumption of sugary drinks, alcohol, and tobacco. A third of South Asia's population is now classified as obese. ${ }^{1}$ These trends contribute to a "risk transition" and a subsequent rise in diabetes mellitus. As most of the T2DM subjects are obese, BMI is an important confounding factor in the association of AN with T2DM. Until now, there is no comprehensive study in the literature evaluating, whether AN is truly related to the T2DM or BMI. In this study, after taking into consideration of various anthropometric measurements and body composition factors in the regression analysis, a significant correlation was seen only with BMI and T2DM.

AN can develop at any site on the skin, most commonly the neck followed by axillae, elbows, knuckles, inner surface of thighs, and popliteal fossa. Previous studies have shown that the severity of AN at the neck correlates with fasting plasma insulin concentrations and BMI. ${ }^{6}$ Although standard dermatological textbooks classify AN into several subtypes, traditionally it can be subdivided into three types: (1) idiopathic form in healthy young children, (2) paraneoplastic form in association with internal malignancy, and (3) AN in obese patients with or without apparent endocrine disorders (previously known as pseudoacanthosis nigricans). The probable pathogenesis of AN in insulin resistance syndromes is attributable to high levels of insulin activating the fibroblasts (dermal cells) and keratinocytes (epidermal cells) via insulin-like growth factor receptors present on these cells. As a result, there will be increased dermal deposition of glycosaminoglycan by fibroblasts leading to papillomatosis, hyperkeratosis and acanthosis because of keratinocyte proliferation.

Previous studies have reported greatly varied prevalence of AN in different ethnic groups, black people being more commonly affected compared with white people. ${ }^{4}$ Stuart et al ${ }^{4}$ for the first time reported the prevalence of $\mathrm{AN}$ in an unselected population, varying from $7 \%$ and $30 \%-40 \%$ in adolescents and adults respectively. Burke et al reported this figure to be $41.1 \%$ in diabetic patients and $31.6 \%$ in healthy subjects. Similarly, we found significantly higher prevalence of AN among diabetic patients (62.6\%) when compared with healthy subjects $(40 \%)$. Apart from the higher prevalence, diabetic patients had more severe grades of AN when compared with healthy subjects. The higher prevalence of AN both in diabetic as well as healthy subjects in our study can be explained by the ethnic predisposition ${ }^{4}$ and the presence of more number of obese subjects in our study groups. The highest prevalence of AN (74\%) was reported by Hud et $a l^{5}$ in a subset of obese population. The prevalence rate of AN among our obese subjects was $72 \%$ and $52.2 \%$ in diabetes and healthy subjects respectively. Consistent with the previous studies, ${ }^{4}$ we found no significant difference in the prevalence of AN among men (48.7\%) and women $(51.4 \%)$. Stuart et $a l^{15}$ reported lower prevalence of $\mathrm{AN}$ in people over 50 years, whereas no such age related difference was found in our study. Similar to the previous studies ${ }^{6}{ }^{16}$ the nape of the neck was the most commonly affected site for AN in our subjects $(93.5 \%)$. 
Like previous studies ${ }^{6}$ we saw significant univariate association of AN with BMI (both in cases and controls), and T2DM. In addition, we noticed a similar significant association of AN with other anthropometric measurements like weight, WC, HC, WHR, and SFT both in cases as well as controls. However, in multivariate regression analysis, presence of AN showed significant correlation only with BMI and T2DM. Regarding severity of AN, a significant difference in various anthropometric measurements (BMI, WC, body fat, and SFT) was seen more clearly between higher grades (grade 2 and grade 3 ) but not in lower grades (grade 1 ).

Body fat percentage calculated with IP was not comparable with that calculated from SFT and this could be attributable to the higher visceral fat in Indians, ${ }^{17}$ which was not measurable with SFT. Furthermore, this discrepancy was even higher in patients with diabetes when compared with healthy subjects because of the higher WC of diabetic patients. Controversy exists between plasma insulin concentrations and risk of developing AN, ${ }^{18}$ however we have not studied this relation in our groups of patients. It is difficult to compare all our results with other studies, as there was no similar study in the literature considering anthropometric measurements and body composition factors in adults with regard to AN in Indians. Thus, these findings have led us to consider that AN is independently associated with BMI and T2DM.

To conclude, Indians have higher prevalence of $\mathrm{AN}$, and it is a cutaneous marker of both T2DM and BMI independent of each other.

\section{Authors' affiliations}

N P Grandhe, S Dogra, B Kumar, Department of Dermatology, Postgraduate Institute of Medical Education and Research, Chandigarh, India

A Bhansali, Department of Endocrinology, Postgraduate Institute of Medical Education and Research, Chandigarh

Funding: none.

Conflicts of interest: none.

\section{REFERENCES}

1 King H, Aubert RE, Herman WH. Global burden of diabetes, 1995-2025: prevalence, numerical estimates, and projections. Diabetes Care 1998;21:1414-31.

2 International Diabetes Federation. Diabetes Atlas, 2000. Brussels: IDF, 2000

3 Gilkison C, Stuart CA. Assessment of patients with acanthosis nigricans skin lesion for hyperinsulinemia, insulin resistance and diabetes risk. Nurse Pract 1992; 17:26, 28, 37 passim.

4 Stuart CA, Pate CJ, Peters EJ. Prevalence of acanthosis nigricans in an unselected population. Am J Med 1989;87:269-72.

5 Hud JA Jr, Cohen JB, Wagner JM, et al. Prevalence and significance of acanthosis nigricans in an adult obese population. Arch Dermatol 1992; 128:941-4.

6 Burke JP, Hale DE, Hazuda HP, et al. A quantitative scale of acanthosis nigricans. Diabetes Care 1999;22:1655-9.

7 The Expert Committee on the Diagnosis and Classification of Diabetes Mellitus. Report of the Expert Committee on the Diagnosis and Classification of Diabetes Mellitus. Diabetes Care 2003;26:S5-20.

8 Stals H, Vercammen C, Peeters C, et al. Acanthosis nigricans caused by nicotinic acid: case report and review of the literature. Dermatology 1994; 189:203-6.

9 Coates $\mathbf{P}$, Shuttleworth D, Rees A. Resolution of nicotinic acid-induced acanthosis nigricans by substitution of an analogue (acipimox) in a patient with type $V$ hyperlipidaemia. Br J Dermatol 1992;126:412-14.

10 Teknetzis A, Lefaki I, Joannides D, et al. Acanthosis nigricans-like lesions after local application of fusidic acid. J Am Acad Dermatol 1993;28:501-2.

11 Tromovitch TA, Jacobs PH, Kern S. Acanthosis nigricans-like lesions from nicotinic acid. Arch Dermatol 1964;89:222-3.

12 Curth HO. Acanthosis nigricans following use of oral contraceptives. Arch Dermatol 1975;111:1069.

13 Snehalatha C, Viswanathan V, Ramachandran A. Cut-off values for normal anthropometric variables in asian Indian adults. Diabetes Care 2003;26:1380-4

14 Siri WE. Body composition from fluid spaces and density: analysis of methods. In: Brozek J, Henschel A, eds. Techniques for measuring body composition. Washington, DC: National Academy of Science, 1961:223-44.

15 Stuart CA, Smith MM, Gilkison CR, et al. Acanthosis nigricans among Native Americans: an indicator of high diabetes risk. Am J Public Health 1994;84: 1839-42.

16 Stuart CA, Gilkison CR, Smith MM, et al. Acanthosis nigricans as a risk factor for non-insulin dependent diabetes mellitus. Clin Pediatr (Phila) 1998:37:73-9.

17 Banerii MA, Faridi N, Atluri R, et al. Body composition, visceral fat, leptin, and insulin resistance in Asian Indian men. J Clin Endocrinol Metab 1999;84:137-44

18 Hirschler V, Aranda C, Oneto A, et al. Is acanthosis nigricans a marker of insulin resistance in obese children? Diabetes Care 2002;25:2353. 